\title{
Sonographic Prediction of Scar Dehiscence in Women with Previous Cesarean Section
}

\author{
Sharma Chanderdeep - Surya Mukesh - Soni Anjali • \\ Soni Pawan Kumar • Verma Ashok • Verma Suresh
}

Received: 6 March 2014/ Accepted: 22 September 2014/Published online: 4 November 2014

(C) Federation of Obstetric \& Gynecological Societies of India 2014

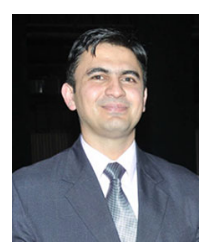

\begin{abstract}
About the Author
Dr. Chanderdeep Sharma is working as an Assistant Professor in the Department of OBG, Dr. Rajendra Prasad Government Medical College, Kangra (H.P.), India, since 2014. He completed his MD (OBG) from Post Graduate Institute of Medical Education and Research Chandigarh in 2011 and received silver medal (first order). He has also received Diplomat of National Board (DNB) from National Board of Examinations in 2012. He completed tenure of senior resident (OBG) from 2011 to 2014 in Dr Rajendra Prasad Government Medical College, Kangra at Tanda (HP), India. He has received "Harold A. Kaminetzsky Prize" for best non-US research paper from American College of Obstetrics and Gynecology (ACOG) in the year 2013. He has eighteen international publications in international indexed journals in short span of 3 years of research. He has also received National Talent Search Examination (NTSE) award. His special interest is in high-risk obstetrics
\end{abstract}

\begin{abstract} vaginal sonography (TVS).

Sharma C. $(\square)$, Assistant Professor (OBG) .

Surya M., Assistant Professor (Radio-diagnosis) .

Soni A., Associate Professor (OBG) .

Soni P. K., Assistant Professor (Radio-diagnosis) .

Verma A., Assistant Professor (OBG) .

Verma S., Professor \& Head of Department of OBG

Dr. RPGMC Kangra, Tanda 176001, HP, India

e-mail: cdsharma2006@gmail.com
\end{abstract}

Purpose To estimate the risk of uterine dehiscence/rupture in women with previous cesarean section (CS) by comparing the thickness of lower uterine segment (LUS) and myometrium with trans-abdominal (TAS) and trans-

Method In this case-control study, in 100 pregnant women posted for elective CS (with or without previous CS; group 1 and group 2 respectively), the thickness of LUS and myometrium was measured sonographically (TAS and TVS). Intra-operatively, LUS was graded (grades I-IV), and its thickness was measured with calipers. The primary outcome of the study was correlation between echographic measurements (TAS and TVS) and features of LUS (grades I-IV) at the time of CS. Secondary outcomes were correlation between myometrial thickness, number of previous CS, and inter-delivery interval with LUS (grades I-IV).

Results Sonographic measurements of LUS and myometrium were significantly different between the two groups (both TAS and TVS $p$ value $=0.000$ each). However, the number of previous CS $(p=0.440)$ and interdelivery interval ( $p=0.062$ ) had no statistically significant correlation with thickness of LUS.

Conclusions Sonographic evaluation of LUS scar and myometrial thickness (both with TAS and TVS) is a safe, reliable, and non-invasive method for predicting the risk of 
scar dehiscence/rupture. Specific guidelines for TOLAC, after sonographic assessment of women with previous CS, are need of the hour.

Keyword Transabdominal ultrasonography . Transvaginal ultrasonography $\cdot$ Cesarean section Pregnancy · Cesarean scar

\section{Introduction}

Cesarean section (CS) rates are increasing worldwide [13]. As a result, women presenting with pregnancy with previous CS are also rising. Previous CS is becoming the most common indication for CS [1], confirming the age old dictum proposed by Edward Craigin in 1914 "Once a cesarean always a cesarean." Although the absolute risk of uterine dehiscence/rupture in lower segment CS is very low $(0.2-1.5 \%)$ [4], the unpredictable nature of this complication and its grave consequences for both mother and baby has resulted in decreased rates of trial of labor after CS (TOLAC) in many countries [5].

Ultrasound estimation of lower uterine segment (LUS) provides a fairly simple and non-invasive method for prediction of scar dehiscence/rupture. The successful outcome of TOLAC depends on the scar of previous CS, which is directly related to its thickness [6]. Evaluation of thickness of LUS has been found to be a potential factor for predicting scar dehiscence [7]. The risk of scar dehiscence/rupture has been directly related to the thinning of LUS [8]. However, there is controversy over the thickness of LUS above which TOLAC can be offered safely [6, 8-10]. Also, Asakura et al. have proposed myometrial thickness as an alternative to LUS thickness for predicting the risk of scar dehiscence/ rupture [11].

Hence, the present study was planned to estimate the risk of scar dehiscence/rupture by ultrasound (TAS and TVS), to determine the correlation between LUS thickness measured by TAS and TVS with actual thickness measured during surgery and to predict an optimal thickness of LUS and myometrium above which women can be safely offered TOLAC.

\section{Materials and Methods}

We conducted an observational study for estimating the risk of scar dehiscence/rupture by sonographic evaluation (TAS and TVS) in women with previous CS. This study was conducted in the Department of Obstetrics and Gynaecology in close collaboration with the Department of Radio-diagnosis of Dr. Rajendra Prasad Government
Medical College and Hospital, Tanda Kangra, India, which is a tertiary care teaching hospital. The recruitment took place from June 2013 to December 2013 after obtaining the approval from the Dr. Rajendra Prasad Government Medical College and Hospital institutional ethics committee. The trial was also registered on the trial registry of India (vide number CTRI/2013/04/ 004991).

All women posted for elective CS were approached for enrollment. Women were eligible for inclusion if they had singleton pregnancy between 36 and 41 weeks of period of gestation and were planned for elective CS. Exclusion criteria were active labor, multiple pregnancy, low lying placenta, leiomyoma in LUS of uterus, previous classical cesarean section/hysterotomy, previous uterine surgery other than CS (myomectomy, hysterotomy, polypectomy, lysis of uterine synechia, or hysteroscopic metroplasty).

Written informed consent was obtained from all the participating women. Previous records were reviewed regarding type of uterine incision and single versus double layered uterine closure. Subsequently, women were divided into two groups. Group 1 consisted of women with at least previous one CS (previous low segment CS with double layered uterine closure; with or without previous vaginal delivery) and Group 2 included women with no previous uterine scar and up to three normal vaginal deliveries (posted for elective CS in this pregnancy as per obstetrical indication).

All these women underwent ultrasound evaluation of the LUS one day prior to the scheduled surgery. All the sonographic measurements were done by the same skilled sonologist (M.S.). Examinations were performed with a scanner (GE Logic P5, GE Healthcare) consisting of a trans-abdominal convex array transducer with a frequency of $3 \mathrm{MHz}$ and a trans-vaginal probe with a frequency of $8 \mathrm{MHz}$. The thickness of the LUS and of its myometrial component was assessed by a sonogram perpendicular to the uterine wall, according to the technique proposed by Jastrow et al. [9]. To measure the thickness of LUS, a cursor was positioned at the interface between the uterine and the bladder wall and another cursor between the amniotic fluid and the deciduas [9].The myometrial thickness was measured with the cursor at the interface of the bladder wall and the myometrium so that it included only the hypoechogenic layer. Three different values of LUS and myometrial thickness were taken, and the lowest value of these was considered as the actual thickness of LUS and the myometrial thickness. To optimize the measurement of LUS, the distension of the bladder was done by a standardized procedure according to Bujold et al. [12]. Women were instructed to empty their bladder and then drink $300 \mathrm{ml}$ of water $1 \mathrm{~h}$ before the examination. If during the 
ultrasound examination uterine contraction was observed, the examination was stopped and resumed after the contraction had subsided. Sonography was also done from the lateral aspect of LUS to detect any asymptomatic dehiscence. Any funneling, ballooning, or wedge defect was noted. Age, parity, gestational age, and neonatal birth weight were assessed for all the women.

At the opening of the abdominal wall during CS, surgeon made an objective evaluation of the integrity and thickness of the LUS, as described by Qureshi et al. [13]. The LUS was graded as follows: Grade I (LUS was well developed), Grade II (LUS was thin without visible content), Grade III (LUS was translucent with visible content), and Grade IV (LUS had well-circumscribed defects, either dehiscence or rupture). All the surgeries were performed by one surgeon (C.D.) to rule out interobserver variation in the assessment of LUS. The operating surgeon was blinded to the sonographic evaluation of the LUS and myometrium.

LUS was identified as the part of the uterus below the loose reflection of the vesico-uterine serosa. After the delivery of neonate, two Green-Armytage forceps were used to hold the lower flap of the uterine incision about 2 inches apart on either side of the midline. The flat upper end of a grasping forceps was placed on the inner aspect of the LUS between the two Green-Armytage forceps to demarcate the inner surface of the LUS. A sterile caliper was placed on the lower flap of the incision at a right angle to the surface of the grasping forceps, and the measurement was taken at three different places, one centimeter apart each, and lowest value was taken as the thickness of the LUS.

Based on the previous studies [13-16], a sample size of 34 women in each group was required to compare the difference in mean LUS thickness, for an $\alpha$ of 0.05 and a power of 0.80 , with an anticipated difference in mean LUS thickness of $0.4 \mathrm{~mm}$ and an anticipated SD of $0.8 \mathrm{~mm}$. Considering $10 \%$ rate of spontaneous labor before surgery and any loss to follow up, a total of 50 women were selected in each group.

Statistical analysis was performed by SPSS 17 software for Windows, using parametric and non-parametric tests when appropriate. The normality of the distribution was assessed by the Kolmogorov-Smirnov test. Continuous data were analyzed with the $t$ test, and categorical variables were analyzed with the Fisher's exact test, when appropriate. $p<0.05$ was considered statistically significant. Receiver operating characteristic (ROC) curve was assessed for the thickness of LUS and myometrium by TAS and TVS in women in group 1. Group 1 was further evaluated for estimating the correlation between myometrial thickness, number of previous CS and inter-delivery interval with LUS (grades I-IV). Further, the correlation of
TAS and TVS with actual thickness of LUS was also determined.

\section{Results}

One hundred and seven women were eligible for inclusion in the study. Three women in group 1 and four women in group 2 went into spontaneous labor before elective CS and as per study protocol were excluded from the study. So, there were 50 women in each group. As shown in Table 1, there was no significant difference in the parity (abortion or previous vaginal delivery), gestational age, neonatal birth weight, or sex of the neonate in either group. However, there was significant difference in the maternal age $27.6 \pm 2.77$ versus $25.1 \pm 4.01$ years (mean $\pm \mathrm{SD}$ : $p=0.000)$ and indications for CS $(p=0.000)$ in two groups.

In group 1, $76 \%$ women had previous one CS only $(n=38), 20 \%$ women had previous two cesarean sections $(n=10)$, and $4 \%(n=2)$ had both previous CS and vaginal delivery.

As shown in Table 2, there were $14 \% \quad(n=7)$ women with early conception ( $<18$ months of inter-delivery interval), $10 \%(n=5)$ had inter-delivery interval of $18-24$ months, $20 \%(n=10)$ had interval of $25-36$ months, and $56 \%(n=28)$ had $>36$ months of inter-delivery interval, in group 1 . We did not correlated the effect of maternal age on grades of LUS as maternal age was significantly different in two groups ( $p$ value $=0.000$ ), and none of the women in group 2 had abnormal grades of LUS (II-IV).

Table 1 Demographic characteristics of women

\begin{tabular}{llll}
\hline & $\begin{array}{l}\text { Group 1 } \\
(n=50)\end{array}$ & $\begin{array}{l}\text { Group 2 } \\
(n=50)\end{array}$ & $p$ value \\
\hline $\begin{array}{l}\text { Maternal age }{ }^{\mathrm{a}} \text { (in years) } \\
\text { Parity }\end{array}$ & $27.6 \pm 2.77$ & $25.1 \pm 4.01$ & 0.000 \\
Abortion & 8 & 5 & 0.372 \\
$\begin{array}{l}\text { Previous vaginal delivery } \\
\text { Gestational age }{ }^{\mathrm{a}} \text { (in }\end{array}$ & 2 & 4 & 0.185 \\
$\quad$ weeks) & & $39.5 \pm 0.13$ & 0.076 \\
$\begin{array}{l}\text { Indication for LSCS } \\
\text { Cephalo-pelvic }\end{array}$ & 23 & & \\
$\quad$ disproportion & & 9 & 0.000 \\
Previous two LSCS & 10 & 0 & \\
Early conception & 7 & 0 & \\
Malpresentation & 10 & 41 & 0.127 \\
Birth weight & & \\
Sex of baby & $3.04 \pm 0.34$ & $2.89 \pm 0.59$ & \\
Boy & & & \\
Girl & 27 & 32 & \\
\hline
\end{tabular}

${ }^{\text {a }}$ Mean \pm standard deviation 
Table 2 Inter-delivery interval from last CS in group 1 and correlation with intra-operative grades II, III, and IV of LUS

\begin{tabular}{|c|c|c|c|c|c|}
\hline Inter-delivery interval (months) & Number of women $(n)$ & Grade II & Grade III & Grade IV & $p$ value \\
\hline$<18$ & $14 \%(n=7)$ & 0 & 1 & 1 & \multirow[t]{4}{*}{0.062} \\
\hline $18-24$ & $10 \%(n=5)$ & 5 & 1 & 1 & \\
\hline $25-36$ & $20 \%(n=10)$ & 4 & 0 & 0 & \\
\hline$>36$ & $56 \%(n=28)$ & 2 & 0 & 0 & \\
\hline
\end{tabular}

Table 3 Intra-operative grades of LUS in women

\begin{tabular}{llllll}
\hline & Grade 1 & Grade 2 & Grade 3 & Grade 4 & $p$ value \\
\hline Group 1 $(n=50)$ & 35 & 11 & 2 & 2 & 0.001 \\
Group 2 $(n=50)$ & 50 & - & - & - & \\
\hline
\end{tabular}

In group $1,80 \%(n=40)$ women had previous one CS and $20 \%(n=10)$ had previous two cesarean sections. In group 2, $82 \%(n=41)$ women were primae gravida, $2 \%$ $(n=1)$ had previous one vaginal delivery, $6 \%(n=3)$ had previous two vaginal deliveries, and remaining $10 \%$ ( $n=5$ ) had abortions ( $n=4$; one abortion and $n=1$; two abortions) prior to this pregnancy.

As shown in Table 3, all women in group 2 had grade I LUS as observed intra-operatively. However, in group 1, thirty-five women had grade I, eleven women had grade II, and two women each had grade III \& IV ( $p$ value = 0.001). None of the women had any funneling, ballooning, or wedge defect in the cesarean scar.

The thickness of LUS and myometrium as measured by TAS and TVS was significantly different ( $p$ value $=0.000$ each) in group 1 and 2, respectively [TAS LUS: $3.96 \pm 0.88$ and $4.80 \pm 1.08 \mathrm{~mm}$, and TVS LUS $4.0 \pm 0.82$ and $4.93 \pm 1.16 \mathrm{~mm}$, TAS myometrium $2.11 \pm 0.42$ and $2.62 \pm 0.66 \mathrm{~mm}$, and TVS myometrium $2.08 \pm 0.53$ and $2.72 \pm 0.70 \mathrm{~mm}$, respectively (mean $\pm \mathrm{SD}$ )], as shown in Table 4 .
Table 5 Correlation between sonographic mean value of LUS and myometrial thickness as measured by TAS and TVS, and number of previous CS with respect to intra-operative grades of LUS

\begin{tabular}{lll}
\hline Correlation & $\begin{array}{l}\text { LUS } \\
\text { (intra-operative grade) }\end{array}$ & $p$ value \\
\hline TAS LUS (Group 1) & I \& II and III \& IV & 0.000 \\
TAS Myometrium (Group 1) & I \& II and III \& IV & 0.000 \\
TVS LUS (Group 1) & I \& II and III \& IV & 0.000 \\
TVS Myometrium (Group 1) & I \& II and III \& IV & 0.029 \\
Number of previous CS (Group 1) & I \& II and III \& IV & 0.440 \\
\hline
\end{tabular}

There was significant correlation $(p$ value $=0.000)$ between LUS and myometrial thickness by TAS and TVS in women of group 1, with surgical LUS grade I \& II with those of grade III \& IV, as shown in Table 5. Furthermore, there was no significant correlation between number of previous CS with surgical LUS grade I \& II and grade III \& IV, in group 1 ( $p=0.440)$.

Figure 1 and 2 shows the ROC curves of TAS LUS and TVS LUS and TAS myometrium (TAS MYO) and TVS myometrium (TVS MYO), respectively. As shown in Table 5, the cutoff value of LUS as measured by TAS was $3.65 \mathrm{~mm}$ (sensitivity $91 \%$, specificity $93 \%$, PPV $14 \%$, NPV $91.2 \%$ ), TVS LUS was $4.05 \mathrm{~mm}$ (sensitivity $71 \%$, specificity $93 \%$, PPV $64 \%$, NPV $95 \%$ ), TAS myometrium was $2.15 \mathrm{~mm}$ (sensitivity $57 \%$, specificity $93 \%$, PPV $59 \%$, NPV $92 \%$ ), and TVS myometrium (sensitivity $65 \%$, specificity $73 \%$, PPV $30 \%$, NPV $92 \%$ ).

Table 4 Difference between mean thickness values of LUS and myometrial thickness by TAS and TVS

\begin{tabular}{llll}
\hline & Group $(n=50)$ & Mean $\pm \mathrm{SD}^{\mathrm{a}}(\mathrm{mm})$ & Mean difference \\
\hline Trans-abdominal sonography & & & 0.84 \\
Total LUS thickness & Group 1 & $3.96 \pm 0.88$ & \\
& Group 2 & $4.80 \pm 1.08$ & 0.51 \\
Total myometrial thickness & Group 1 & $2.11 \pm 0.42$ & 0.000 \\
& Group 2 & $2.62 \pm 0.66$ & 0.87 \\
Trans-vaginal sonography & & & 0.000 \\
Total LUS thickness & Group 1 & $4.06 \pm 0.82$ & 0.64 \\
& Group 2 & $4.93 \pm 1.16$ & 0.000 \\
Total myometrial thickness & Group 1 & $2.08 \pm 0.53$ & 0.000 \\
& Group 2 & $2.72 \pm 0.70$ & \\
\hline
\end{tabular}

${ }^{\mathrm{a}}$ Mean \pm standard deviation 
Fig. 1 ROC curves showing TAS and TVS assessment of LUS. Diagonal segment are produced by ties. TAS transabdominal sonography, LUS lower uterine segment thickness, TVS trans-vaginal sonography
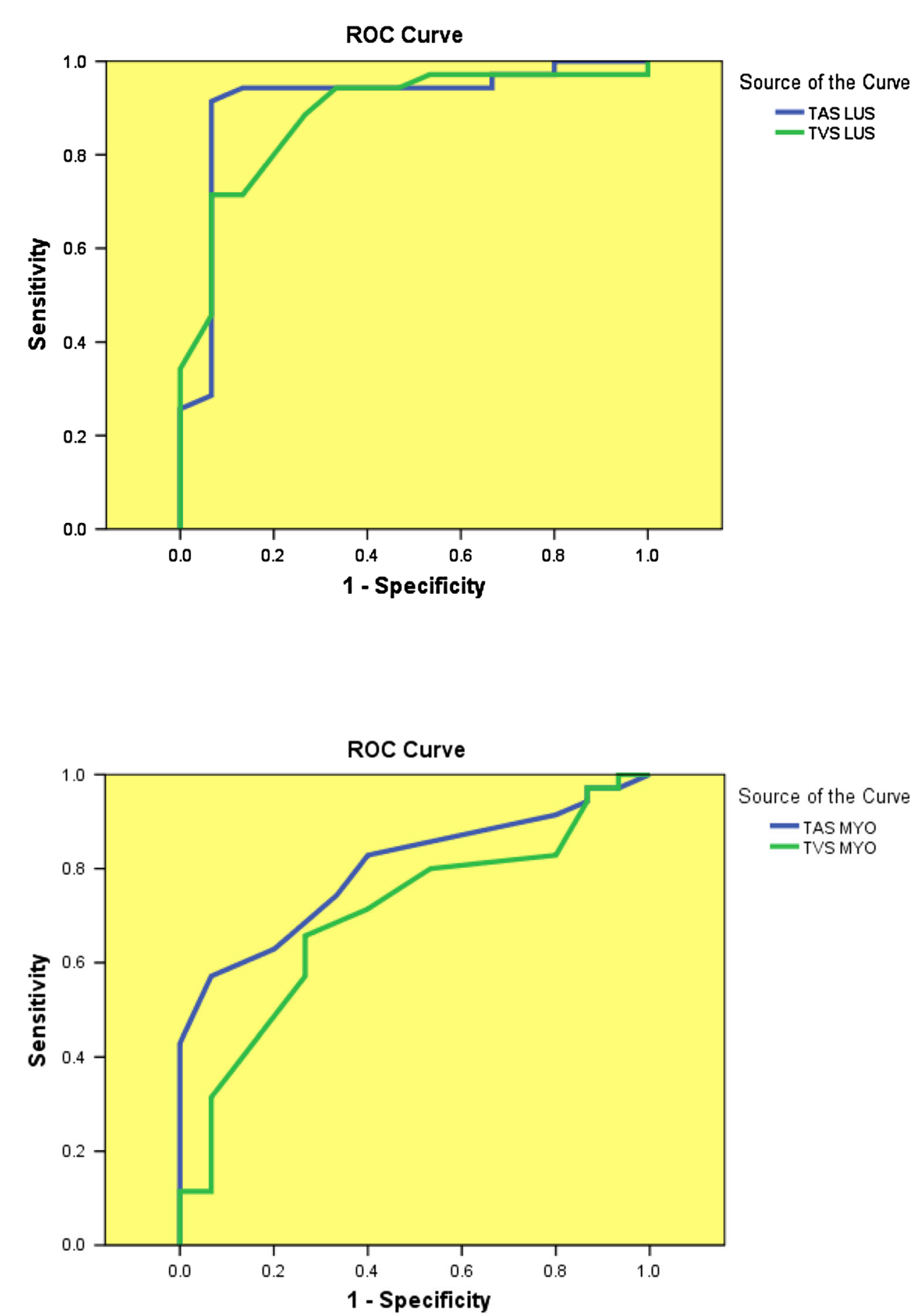

Fig. 2 ROC curves showing trans-abdominal and transvaginal sonographic assessment of myometrial thickness. Diagonal segment are produced by ties TAS trans-abdominal sonography, MYO myometrial thickness, TVS trans-vaginal sonography . 
( $p=0.000)$ in women with LUS grades I \& II and III \& IV, as shown in Table 5. We observed that the LUS thickness less than $3.65 \mathrm{~mm}$ has $91 \%$ sensitivity, $93 \%$ specificity, and $91 \%$ negative predictive value (Table 5; Fig. 2; ROC curve) as measured by TAS. Rozenberg et al. [8] and Jastrow et al. [16] had proposed cut off of $3.5 \mathrm{~mm}$ for LUS thickness above which VBAC can be safely offered to women with previous CS. We also observed that in all women with LUS grades III \& IV, LUS thickness (as measured by TAS and TVS) was less than $3 \mathrm{~mm}$. Hence we propose, $3.65 \mathrm{~mm}$ of LUS thickness as measured by TAS to be a safe limit above which VBAC can be safely offered.

Sonographic measurement of thickness of myometrium has been analyzed by Asakura et al. [11] and Gizzo et al. [10]. We observed statistically significant correlation between thickness of myometrium as measured by TAS and TVS $(p=0.000)$ in women with LUS grades I \& II and III \& IV, (as shown in Table 5). Thickness of myometrium less than $2.15 \mathrm{~mm}$ has $57 \%$ sensitivity, $93 \%$ specificity, and $92 \%$ negative predictive value (Table 5, Fig. 2; ROC curve) as measured by TAS. So, we propose that thickness of myometrium (cut off $2.15 \mathrm{~mm}$ ) should be used as an adjunct to the LUS thickness for estimating the catastrophic outcome of scar dehiscence/rupture.

As proposed by American College of Obstetrics and Gynecology [14], women with previous two CS can reasonably be offered TOLAC. In our study, women with previous two CS (in group 1) when compared with respect to LUS grades I \& II and III \& IV between themselves (as shown in Table 5) had no statistically significant difference ( $p$ value $=0.440)$, similar to observation of Gizzo et al. [10].

One of the major deterrents for offering TOLAC in women with previous one CS is early conception/short interpregnancy interval [14]. In our study, women with interdelivery interval less than 18 months $(n=7 / 50)$, when compared with other women in group 1 with respect to LUS grades II-IV, approached significance $(p=0.062)$. This observation is contrary to the observation of other investigators $[10,14]$. This could be probably due to less number of women $(n=7)$ with short inter-delivery interval in group 1 .

Our study further confirms the available evidence regarding the usefulness and efficacy of sonographic evaluation of LUS and myometrium for safely predicting the outcome of TOLAC. Also it re-affirms the view of ACOG regarding TOLAC in women with previous two cesarean sections, as the benefits of successful outcome of TOLAC in terms of maternal and neonatal benefits is well documented. Although the correlation between interdelivery interval and grades of LUS (II-IV) approached significance $(p=0.062)$, it was not statistically significant probably due to less number of women $(n=7)$. A definite technique for use of ultrasound for measurement of LUS and myometrium needs to be standardized (as per Jastrow et al. [9] ), filling of urinary bladder before sonography (Bujold et al. [12]), and grades of LUS (Qureshi et al. [13]), and training module for obstetricians and sonologists should be offered for assessment of LUS and myometrium.

Observations of present study corroborate the fact that sonographic measurement of thickness of LUS and myometrium is an excellent method for safely predicting the risk of scar dehiscence/rupture in women with previous CS. With different cut off for critical values of thickness of LUS and myometrium in different studies [8-11], specific guidelines regarding the measurement technique of LUS by TAS and TVS, critical thickness of LUS and myometrium for evaluation of LUS is need of the hour to save many pregnant women and babies from significant morbidity and mortality.

Compliance with ethical requirements and Conflict of interest Ethical clearance has been taken from ethical committee of Dr. RPGMC Kangra at Tanda (HFW-H-DRPGMC/Ethics/2013/22 dated 13th April 2013) keeping in view the ICMR guidelines (1994) and Helinski declaration (modified 2000). The trial was also registered in Clinical trial registry of India (CTRI/2013/04/004991). Sonography is a routine procedure in obstetric patients. Written informed consent was taken from all the women. Identity of every women is kept as secret. All the women were adequately informed of the aims, methods, and any discomfort it may entail to her and the remedies thereof. Every precaution was taken to respect the privacy of the patient, the confidentiality of the patient's information, and to minimize the impact of the study on her physical and mental integrity and her personality. The patient was given the right to abstain from the study or to withdraw consent to participate at any time of study without reappraisal. The authors declare that they have no conflict of interest.

\section{References}

1. Chanrachakul B, Herabutya Y, Udomsubpayakul U. Epidemic of cesarean section at the general, private and university hospitals in Thailand. J Obstet Gynaecol Res. 2000;26(5):357-61.

2. Villar J, Valladares E, Wojdyla D, et al. Ceasarean delivery rates and pregnancy outcomes: the 2005 world global survey on maternal and perinatal health in Latin America. Lancet. 2006;367(9525):1819-29.

3. Devendra K, Arulkumaran S. Should doctors perform an elective caesarean section on request? Ann Acad Med Singap. 2003;32(5):577-81.

4. Cheung VY, Constantinescu OC, Ahluwalia BS. Sonographic evaluation of the lower uterine segment in patients with previous cesarean delivery. J Ultrasound Med. 2004;23:1441-7.

5. Yeh J, Wactawski-Wende J, Shelton JA, et al. Temporal trends in the rates of trial of labor in low-risk pregnancies and their impact on the rates and success of vaginal birth after cesarean delivery. Am J Obstet Gynecol. 2006;194(1):144.

6. Sen S, Malik S, Salhan S. Ultrasonographic evaluation of lower uterine segment thickness in patients of previous cesarean section. Int J Gynecol Obstet. 2004;87(3):215-9.

7. Ofili-Yebovi D, Ben-Nagi J, Sawyer E, et al. Deficient lowersegment Cesarean section scars: prevalence and risk factors. Ultrasound Obstet Gynecol. 2008;31(1):72-7. 
8. Rozenberg P, Goffinet F, Phillippe HJ, et al. Ultrasonographic measurement of lower uterine segment to assess risk of defects of scarred uterus. Lancet. 1996;347:281-4.

9. Jastrow N, Chaillet N, Roberge S, et al. Sonographic lower uterine segment thickness and risk of uterine scar defect: a systematic review. J Obstet Gynaecol Can. 2010;32:321-7.

10. Gizzo S, Zambon A, Saccardi C, et al. Effective anatomical and functional status of the lower uterine segment at term: estimating the risk of uterine dehiscence by ultrasound. Fertil Steril. 2013; 99(2):496-501.

11. Asakura H, Nakai A, Ishikawa G, et al. Prediction of uterine dehiscence by measuring lower uterine segment thickness prior to the onset of labor: evaluation by transvaginal ultrasonography. J Nippon Med Sch. 2000; 67:352-6.

12. Bujold E, Jastrow N, Simoneau J, et al. Prediction of complete uterine rupture by sonographic evaluation of the lower uterine seg-ment. Am J Obstet Gynecol. 2009;201:320.
13. Qureshi B, Inafuku K, Oshima K, et al. Ultrasonographic evaluation of lower uterine segment to predict the integrity and quality of cesarean scar during pregnancy: a prospective study. Tohoku J Exp Med. 1997;183:55-65.

14. American College of Obstetricians and Gynecologists. ACOG practice bulletin no. 115: vaginal birth after previous cesarean delivery. Obstet Gynecol. 2010;116(2):450-63.

15. Cheung VY. Sonographic measurement of the lower uterine segment thickness in women with previous caesarean section. J Obstet Gynaecol Can. 2005;27(7):674-81.

16. Jastrow N, Antonelli E, Robyr R, et al. Inter- and intraobserver variability in sonographic measurement of the lower uterine segment after a previous Cesarean section. Ultrasound Obstet Gynecol. 2006;27:420-4. 